\title{
DEGREES OF RECURSIVELY SATURATED MODELS
}

\author{
BY
}

\author{
ANGUS MACINTYRE AND DAVID MARKER
}

\begin{abstract}
Using relativizations of results of Goncharov and Peretyat'kin on decidable homogeneous models, we prove that if $M$ is $S$-saturated for some Scott set $S$, and $F$ is an enumeration of $S$, then $M$ has a presentation recursive in $F$. Applying this result we are able to classify degrees coding (i) the reducts of models of PA to addition or multiplication, (ii) internally finite initial segments and (iii) nonstandard residue fields. We also use our results to simplify Solovay's characterization of degrees coding nonstandard models of Th(N).
\end{abstract}

0. Introduction. The classic theorem of Tennenbaum [T] says that there is no recursive nonstandard model of Peano arithmetic. Indeed, if $(\omega, \oplus, \odot) \vDash \mathbf{P}$, and $(\omega, \oplus, \odot)$ is nonstandard, then neither $\oplus$ nor $\odot$ is recursive. In the above situation, $(\omega, \oplus)$ and $(\omega, \odot)$ are recursively saturated, and nowadays one formulates the theorem as the statement that there are no recursive, recursively saturated models of either Presburger or Skolem arithmetic. Let us say a theory $T$ has the Tennenbaum property if $T$ has no recursive, recursively saturated models. Macintyre proved in [Mac1] that most theories of fields have the Tennenbaum property.

In this paper we look at a natural refinement of the Tennenbaum property. For any countable structure $M$ we look at Turing degrees of presentations of $M$. If $M \vDash \mathbf{P}, M$ nonstandard, and $M$ is isomorphic to $(\omega, \oplus, \odot), 0$ is not the Turing degree of $\oplus$, nor of $\odot$. But given $M$, what are the possible degrees for $\oplus, \odot$ ? And how do these relate to the possible degrees for $\langle\oplus, \odot\rangle$ ?

Here is a sample of results proved below:

(a) the set of degrees for $\oplus$ equals the set of degrees for $\odot$;

(b) the set of degrees for $\oplus$ and $\odot$ are closed upwards;

(c) if $M$ is recursively saturated, the set of degrees for $\langle\oplus, \odot\rangle$ is the same as the set of degrees for $\oplus$.

These are corollaries of a very general theorem about recursively saturated models of effectively perfect theories $T$. If $\mathfrak{A} \vDash T, \mathfrak{A}$ recursively saturated, there is associated with $\mathfrak{U}$ a canonical Scott set $S$. Our main theorem relates degrees of presentations of $\mathfrak{A}$ and degrees of presentations of $S$, showing that the sets are mutually dense. The essential tool we use is the Goncharov-Peretyat'kin Theorem on homogeneous models [G, P], suitably relativized. A priority argument is needed here.

Received by the editors November 4, 1982.

1980 Mathematics Subject Classification. Primary 03H15; Secondary 03D45, 03C50.

Key words and phrases. Scott set, Turing degree, recursively saturated model, model of arithmetic. 
We give applications to sharpened Tennenbaum theorems for reducts of nonstandard models $M$ of $\mathbf{P}$. Perhaps most noteworthy is the following:

Let $a \in M$ be nonstandard, and $p$ a nonstandard prime. Then for any degree $d$,

$\oplus_{M}$ has a presentation of degree $d \Leftrightarrow M / p$ has a presentation of degree $d$ $\Leftrightarrow([0, a], \oplus, \odot)$ has a presentation of degree $d$.

Some of the results extend to fragments of $\mathbf{P}$.

The paper originated in observations such as the following:

If $M$ is a countable recursively saturated model of $\mathbf{P}, M$ is uniquely determined by any $M / p, p$ an infinite prime.

We would like to thank George Wilmers and Julia Knight.

1. Scott sets and recursive saturation. A set $X \subseteq 2^{\omega}$ is a tree iff for any $\sigma \in X$ if $\tau \subseteq \sigma$, then $\tau \in X$. We fix a recursive identification of $2^{\omega}$ and $\omega$, and consider trees as subsets of $\omega$.

Definition 1.1. $X \subseteq P(\omega)$ is a Scott set iff

(i) if $a_{1}, \ldots, a_{n} \in X$, and $b$ is recursive in $a_{1}, \ldots, a_{n}$, then $b \in X$ and

(ii) if $T$ is an infinite tree in $X, T$ has a branch coded in $X$.

Scott sets were introduced by Scott $[\mathbf{S}]$ and have been studied extensively by Wilmers [W1, W2] and Knight and Nadel [KN1, KN2 and K1]. Most of the results mentioned in this section can be found in [W2 or KN1]. Throughout the paper we will assume all structures are countable.

Definition 1.2. Suppose $S \subseteq P(\omega)$. M is $S$-saturated iff

(i) every pure type realized in $M$ is recursive in some $s \in S$, and

(ii) if $p(\bar{x}, \bar{y})$ is a pure type recursive in some $s \in S, \bar{m} \in M$, and $p(\bar{x}, \bar{m})$ is consistent, then $p(\bar{x}, \bar{m})$ is realized in $M$.

The following lemma sums up most of the useful facts about $S$-saturation and Scott sets. For $M$ an $L$-structure, Typ $(M)$ denotes the set of (codes of) pure types realized in $M$.

Lemma 1.3. (i) If $M$ is $S$-saturated, then $\operatorname{Typ}(M) \subseteq S$ and $\operatorname{Th}(M) \in S$.

(ii) $M$ is recursively saturated iff $M$ is $S$-saturated for some $S$ cott set $S$.

(iii) If $S$ is a countable Scott set and $T$ is a complete theory recursive in some $s \in S$, then $T$ has a countable $S$-saturated model.

(iv) If $M$ is countable and $S$-saturated, then $M$ is determined up to isomorphism by $S$ and $\operatorname{Th}(M)$.

Proof. (i) is immediate from our definitions.

(ii) Clearly if $M$ is $S$-saturated, then $M$ is recursively saturated. For the converse, let $S=\{d \subseteq \omega$ : if $\bar{m} \in M, \Gamma(\bar{v}, \bar{w})\}$ is consistent with $\operatorname{Th}(M)$, then $\Gamma(\bar{v}, \bar{m})$ is realized in $M$. It can easily be shown that $M$ is $S$-saturated. See [KN1] for details.

(iii) This is proved by a Henkin argument similar to the one used by Scott [S].

(iv) Any two $S$-saturated models of a complete theory are $\omega$-homogeneous and realize the same types.

For many theories, Lemma 1.3(ii) may be significantly strengthened. 
DEFINITION 1.4. $T$ is effectively perfect if for some $n$ there is a map $\varphi$ from $2^{\omega}$ to the set of consistent formulas in $n$-free variables such that:

(i) $\varphi$ is recursive in $T$,

(ii) if $\sigma \subseteq \tau$, then $T \vdash \varphi(\tau) \rightarrow \varphi(\sigma)$, and

(iii) $\varphi(\hat{\sigma 0})$ and $\varphi(\hat{\sigma} \hat{l})$ are incompatible.

EXAMPLES. (i) If $T$ is a complete extension of Presburger arithmetic in any suitable language, then $T$ is effectively perfect. The function $\varphi$ may be given by $\varphi(\alpha)=$ $\mathbb{X}\left\{p_{n} \mid v: \alpha(n)=1\right\} \wedge \mathbb{X}\left\{p_{n} \mid v: \alpha(n)=0\right\}$, where $p_{n}$ denotes the $n$th prime number.

(ii) Macintyre [Mac1] has shown that with the possible exception of some very bizarre cases not known to exist, all theories of infinite fields which are not algebraically closed are effectively perfect.

(iii) Wilmers [W2] and Knight and Nadel [KN1] show that if $T$ is not atomic then $T$ is effectively perfect.

(iv) Knight and Nadel [KN1] observe that if $T$ has an infinite recursive sequence of independent formulas, then $T$ is effectively perfect.

THEOREM 1.5 [KN1, N, W2]. If $T$ is effectively perfect and $M \vDash T$ is recursively saturated, then $M$ is $S$-saturated for a unique Scott set $S$.

Proof. Fix $n$ and $\varphi$ witnessing the fact that $T$ is effectively perfect. Let $\bar{m} \in M^{n}$. We say $\bar{m}$ codes $f \in 2^{\omega}$ iff $M \vDash \varphi(\alpha)(\bar{m})$ for all $\alpha \subseteq f$. Let $S=\{X \subseteq \omega$ : some $\bar{m} \in M^{n}$ codes the characteristic function of $\left.X\right\}$.

Claim. $S$ is a Scott set and $M$ is $S$-saturated.

Suppose $\bar{m}$ codes $f$ and $f_{1} \leqslant_{T} f$. Consider the type $\Gamma(\bar{v})=\left\{\varphi(\beta)(\bar{v}): \beta \subseteq f_{1}\right\}$. By Lemma 1.3(i) and (ii), $M$ must realize $\Gamma(\bar{v})$ by some $\bar{n}$. This $\bar{n}$ codes $f_{1}$. Similar arguments show that $S$ is closed under joins and has the tree property. Lemma 1.3 also implies $M$ is $S$-saturated.

Suppose that $M$ is also $S^{\prime}$-saturated. Then $S^{\prime} \subseteq S$. Let $s^{\prime} \in S^{\prime}$. Coding arguments similar to those above show that $s^{\prime}$ is coded by some $\bar{m} \in M^{n}$. Thus $S^{\prime}=S$.

Knight and Nadel [KN1] show that the conclusion of Theorem 1.5 also holds if $T$ has pure types of every degree. Some condition on $T$ is evidently necessary as algebraically closed fields provide a theory where any recursively saturated model is $S$-saturated for every $S$.

\section{Some effective model theory.}

Definition 2.1. Given an $L$-structure $M$, a presentation of $M$ is a bijection $f$ : $\omega \rightarrow M$. Such an $f$ induces an $L$-structure $M^{f}$ with universe $\omega$ such that $M^{f}$ F $\varphi\left(m_{1}, \ldots, m_{n}\right)$ iff $M \vDash \varphi\left(f\left(m_{1}\right), \ldots, f\left(m_{n}\right)\right)$.

Suppose $N$ is an $L$-structure with underlying set $\omega$, and $\Gamma$ is a set of $L$-formulas. The $\Gamma$-degree, of $N$ is the Turing degree of $\left\{\left\langle\varphi\left(v_{1}, \ldots, v_{n}\right),\left(m_{1}, \ldots, m_{n}\right)\right\rangle\right.$ : $\varphi\left(v_{1}, \ldots, v_{n}\right) \in \Gamma$ and $\left.N \vDash \varphi\left(m_{1}, \ldots, m_{n}\right)\right\}$. If $M$ is an arbitrary structure, $D(\Gamma, M)$ is defined to be the set of $\Gamma$-degrees of $M^{f}$ as $f$ runs through the set of presentations of $M$. 
DefinITION 2.2. Let $X \subseteq 2^{\omega}$ be countable. $f: \omega^{2} \rightarrow 2$ is an enumeration of $X$ iff $X=\{\{n: f(m, n)=1\}: m \in \omega\}$. Let $D(X)=\{d:$ there is an enumeration $f$ of $X$ such that $\left.f \equiv_{T} d\right\}$.

Lemma 2.3. (i) If $d \in D(X)$, then, for any $x \in X, x \leqslant T d$.

(ii) If $X$ is a Scott set and $d \in D(X)$, then for any $x \in X$ any tree recursive in $x$ has a branch recursive in $d$.

(iii) If for some $n \in \omega,\{g \in X: g(n)=1\}$ is infinite and coinfinite then $D(X)$ is upward closed in the Turing degrees.

Proof. (i) and (ii) are obvious.

(iii) Let $n$ be such that $\{g \in X: g(n)=1\}$ is infinite and coinfinite. Let $f$ be an enumeration of $X$ and suppose $d_{T} \geqslant f$. We can easily find $h: \omega \rightarrow \omega$ such that $h$ is a surjection, $h \leqslant_{T} d$ and $f(h(m), n)=1$ iff $n \in d$. Define $F: \omega^{2} \rightarrow 2$ by $F(m, n)=$ $f(h(m), n)$. Clearly $F \leqslant_{T} d$ and $F$ enumerates $X$. But $m \in d$ iff $F(m, n)=1$ so $F \equiv d$.

One might wonder if there is a converse to 2.3(i). Namely, if $S$ is a Scott set and for every $s \in S, d \geqslant s$, then is $d \in D(S)$ ? Knight, Lachlan and Soare [KLS] show that this need not be the case. In their refutation of Knight's conjecture they produce a degree $d$ greater than all arithmetic degrees such that if $E \subseteq \omega^{2}$ is recursive in $d$, then $\{\{n:(m, n) \in E\}: m \in \omega\}$ does not contain all arithmetic degrees. Their proof can be modified to work for any Scott set $S$.

LEMma 2.4. Let $\Delta$ be the set of all L-formulas. Let $M$ be an L-structure. If $d \in D(\Delta, M)$, then $\operatorname{Typ}(M)$ has an enumeration recursive in $d$.

Proof. Let $M^{g}$ have $\Delta$-degree $d$. Let $\sigma: \omega \rightarrow \omega^{\omega}$ and $\tau: \omega \rightarrow L$-formulas be recursive bijections. Define $f: \omega^{2} \rightarrow 2$ such that $f(m, n)=1$ iff $M^{g} \vDash \varphi\left(i_{1}, \ldots, i_{j}\right)$, where $\tau(n)=\varphi\left(v_{1}, \ldots, v_{j}\right)$ and $\sigma(m)=\left\langle i_{1}, \ldots, i_{j}\right\rangle$.

From Lemma 2.4 we conclude that $D(\operatorname{Typ}(M))$ is dense in $D(\Delta, M)$. Thus by Lemma 2.3(iii), $D(\operatorname{Typ}(M)) \supseteq D(\Delta, M)$. The remainder of this section is devoted to showing that under certain hypotheses we may reverse this inclusion.

Definition 2.5. Let $S$ be a set of types. An enumeration $f$ of $S$ has the $d$-effective extension property iff $d_{T} \geqslant f$ and there is a $d$-recursive function $g: \omega^{2} \rightarrow \omega$ such that if $p\left(x_{0}, \ldots, x_{n-1}\right) \in S, p=\left\{\psi: f\left(m,{ }^{\ulcorner} \psi^{\top}\right)=1\right\}$ and $\varphi\left(x_{0}, \ldots, x_{n}\right)$ is consistent with $p$, then $\left\{\psi: f\left(g\left(m,{ }^{\top} \varphi^{\urcorner}\right),{ }^{\ulcorner} \psi^{\top}\right)=1\right\}$ is an extension of $p$ in the variables $x_{0}, \ldots, x_{n}$ containing $\varphi\left(x_{0}, \ldots, x_{n}\right)$.

The following theorem is a relativized version of a result due independently to Goncharov [G] and Peretyat'kin [P], which improves earlier work of Morley [Mo] and Millar [Mi].

THEOREM 2.6. Let $M$ be w-homogeneous. Suppose $f$ is an enumeration of $\operatorname{Typ}(M)$ with the d-effective extension property. If $\Delta$ denotes the set of all L-formulas, then there is an $e \in D(\Delta, M)$ such that $e \leqslant_{T} d$.

Proof. See [P or G].

We will show that the hypothesis of Theorem 2.6 holds reasonably often. 
Definition 2.7. A set $S$ of types is Turing closed iff, for any $p \in S$, if $q$ is a type and $q \leqslant_{T} p$, then $q \in S$.

Suppose $X \subseteq 2^{\omega}$ and $T$ is a complete theory coded by some $x \in X$. Let $\operatorname{Typ}^{n}(X)$ be the set of complete $n$-types over $T$ which are coded in $X$. Of course $\operatorname{Typ}^{n}(X)$ depends on our choice of $T$.

Lemma 2.8. If $\operatorname{Typ}^{n}(X)$ is Turing closed and $f$ is an enumeration of $X$, then $\operatorname{Typ}^{n}(X)$ has an enumeration recursive in $f$.

Proof. We will consider only the case $n=1$. We dovetail over $\omega \times \omega$ to decide longer and longer parts of $\{n: f(m, n)=1\}$ for each $m$. We say that $m$ is active at stage $s$ of our construction iff

(i) $m$ is active at each stage $s^{\prime}, s^{\prime}<s$,

(ii) $\{n \leqslant s: f(m, n)=1\}$ is a set of formulas consistent with $T$, and

(iii) there is no formula $\varphi(v)$ such that ${ }^{\Gamma} \varphi(v)^{\urcorner} \leqslant s$ : ${ }^{\Gamma} \neg \varphi(v)^{\urcorner} \leqslant s$ and $f\left(m,{ }^{\ulcorner} \varphi(v)^{\urcorner}\right)=f\left(m,{ }^{\ulcorner} \neg \varphi(v)^{\urcorner}\right)$.

These conditions are recursive in $T$ and as $T$ is coded in $X$, they can be answered effectively in $f$.

If $m$ is not active at stage $s$, there is a greatest $t<s$ such that $m$ was active at stage $t$. Thus $\Gamma=\left\{\varphi(v):{ }^{\ulcorner} \varphi^{\urcorner} \leqslant t\right.$ and $\left.f\left(m,{ }^{\ulcorner} \varphi^{\urcorner}\right)=1\right\} \cup\left\{\neg \varphi:{ }^{\ulcorner} \varphi^{\urcorner} \leqslant t\right.$ and $f\left(m,{ }^{\ulcorner} \varphi^{\urcorner}\right)$ $=0\}$ codes a set of formulas consistent with $T$. We may now uniformly complete $\Gamma$ by a Henkin process effective in $T$.

We define $g: \omega^{2} \rightarrow 2$ as follows:

$$
g(m, n)=\left\{\begin{array}{l}
f(m, n) \text { if } m \text { is active at stage } n \\
\text { the decision of the Henkin process }
\end{array}\right. \text { otherwise. }
$$

Clearly $g \leqslant_{T} f$. If $p \in \operatorname{Typ}^{\mathrm{l}}(X)$ and $\varphi \in p$ iff $f\left(m,{ }^{\ulcorner} \varphi^{\urcorner}\right)=1$, then $\varphi \in p$ iff $g\left(m,{ }^{\ulcorner} \varphi^{\urcorner}\right)=1$. Also if $q=\left\{\varphi: g\left(m,{ }^{\ulcorner} \varphi^{\urcorner}\right)=1\right\}$, then either:

(i) For every $n, m$ is active at stage $n$. In this case $q$ is a type and $q=\{\varphi$ : $\left.f\left(m,{ }^{r} \varphi^{\top}\right)=1\right\}$, so $q \in \operatorname{Typ}^{1}(X)$, or

(ii) for some $n, m$ is not active at stage $n$. In this case $q \leqslant_{T} T$ and so, since $\operatorname{Typ}^{1}(X)$ is Turing closed, $q \in \operatorname{Typ}^{1}(X)$. Thus $g$ is an enumeration of $\operatorname{Typ}^{1}(X)$.

LEMMA 2.9. Let $T$ be a complete theory. There is a uniform recursive operator $\Psi$ such that if $p$ is a type over $T$ in the variables $x_{0}, \ldots, x_{n-1}$ and $\varphi\left(x_{0}, \ldots, x_{n}\right)$ is a formula consistent with $p$, then $\Psi(p, \varphi)$ is a type in the variables $x_{0}, \ldots, x_{n}$ such that $\Psi(p, \varphi) \supseteq p, \varphi \in \Psi(p, \varphi)$ and $\Psi(p, \varphi) \leqslant_{T} p$.

Proof. $\Psi$ is just the usual Henkin procedure for finding a complete extension of $p$ containing $\varphi$.

THEOREM 2.10. If $S \subseteq w^{\omega}$ is downward closed in the Turing degrees, $f$ is an enumeration of $S$ and $f \leqslant_{T} d$, then $\operatorname{Typ}(S)$ has an enumeration $g$ with the d-effective extension property.

Proof. We inductively define a set $I$ of indices by the rules:

(i) if $n \in \omega$, then $n \in I$, and 
(ii) if $i \in I$ and $\varphi\left(x_{0}, \ldots, x_{n}\right)$ is a formula, then $\langle i, \varphi\rangle \in I$.

Let $f_{0}: \omega^{2} \rightarrow 2$ be an enumeration of $\operatorname{Typ}(S)$ obtained from Lemma 2.8 .

Let $\sigma: \omega \rightarrow I$ be a recursive bijection. We define $g: \omega^{2} \rightarrow 2$ inductively on indices as follows:

(i) if $\sigma(m) \in \omega$, then $g(m, n)=f_{0}(\sigma(m), n)$,

(ii) if $\sigma(m)=\left\langle i, \varphi\left(x_{0}, \ldots, x_{n}\right)\right\rangle, \varphi\left(m_{0}\right)=i$, and $q=\left\{\theta: g\left(m_{0},{ }^{\ulcorner} \varphi^{\top}\right)=1\right\}$ is a type in the variables $x_{0}, \ldots, x_{n-1}$ consistent with $\varphi\left(x_{0}, \ldots, x_{n}\right)$, then $g(m, n)$ is defined to code $\Psi\left(q, \varphi\left(x_{0}, \ldots, x_{n}\right)\right)$,

(iii) otherwise $g(m, n)=f_{0}(0, n)$.

Clearly $g$ is an enumeration of $\operatorname{Typ}(X)$. Let $h: \omega \times \omega \rightarrow \omega$ be defined by $h\left(m,{ }^{\ulcorner} \varphi^{\top}\right)=\sigma^{-1}(\langle\sigma(m), \varphi\rangle) . h$ is easily seen to witness the $d$-effective extension property.

We may now state our partial converse to Lemma 2.4.

THEOREM 2.11. If $M$ is $\omega$-homogeneous, $S$ is the set of degrees of pure types realized in $M, \operatorname{Typ}(M)$ is Turing closed and $f$ is an enumeration of $S$, then $M$ has a presentation recursive in $f$. (Restated: if $\Delta$ is the set of all L-formulas, then $D(\Delta, M)$ is dense in $D(S))$.

Proof. The proof is clear from Lemma 2.9 and Definition 2.5.

Goncharov and Peretyat'kin $[\mathbf{G}, \mathbf{P}]$ have examples which show that we cannot omit the assumption that $\operatorname{Typ}(M)$ is Turing closed. We will be using only the following corollary.

Corollary 2.12. If $M$ is $S$-saturated and $f$ is an enumeration of $S$, then $M$ has presentation recursive in $f$.

3. Applications to arithmetic. (a) Presburger arithmetic. Let $T$ be a complete theory in a language extending $L=\{+, 0,1\}$ such that $T \geqslant \operatorname{Th}(\omega,+, 0,1)$. For $M=T$, $M_{+}$denotes the reduct of $M$ to $L$. For $a \in M$ we define the real coded by $a$, $r(a)=\left\{n \in \omega: p_{n} \mid a\right\}$, where $p_{n}$ denotes the $n$th prime number. $\operatorname{Re}(M)$ denotes $\{r(a): a \in M\}$. Smorynski $[\mathbf{S m}]$ is a good recent reference.

Let $\nabla$ be the set of basic $L$-formulas. Let $d$ be the $\nabla$-degree of $M^{f}$. If $i \in \omega$, we claim that $r(f(i))$ is recursive in $d$. We first note that

$$
n \in r(f(i)) \text { iff } \exists m \in \omega(M^{f} \vDash \underbrace{m+\cdots+m}_{p_{n} \text { times }}=1) .
$$

Thus $r(f(i))$ is r.e. in $d$. On the other hand $p_{n} \nmid a$ iff $\exists y\left(p_{n} y<a<p_{n}(y+1)\right)$. So $n \notin r(f(i))$ iff $\exists y, z, w\left(M^{f}=p_{n} y+w=i \wedge w \neq 0 \wedge i+z=p_{n}(y+1) \wedge z \neq 0\right)$. Thus $r(f(i))$ is recursive in $d$ uniformly in $i$. We also see that $d \in D(\operatorname{Re}(M))$ since

$$
g(m, n)= \begin{cases}1, & M^{f}=p_{n} \mid m, \\ 0, & M^{f}=p_{n} \nmid m,\end{cases}
$$

is an enumeration of $\operatorname{Re}(M)$ recursive in $d$.

Recall from $\S 1$ that $T$ is effectively perfect. Thus by Theorem 1.5 if $M \vDash T$ is recursively saturated, then $M$ is $S$-saturated for a unique Scott set $S$. We will often refer to such an $S$ as the Scott set of $M$. 
LEMMA 3.1. If $M \vDash T, M_{+}$is recursively saturated and $S$ is the Scott set of $M_{+}$, then $S=\operatorname{Re}(M)$.

Proof. We need only show that $M$ is $\operatorname{Re}(M)$-saturated. Let $\bar{a} \in M$ and $\Gamma(v)=$ $\left\{p_{n} \mid v \leftrightarrow \varphi(\bar{a}): \varphi(\bar{v})\right.$ a formula $\}$. As $M$ is recursively saturated $\Gamma$ is realized in $M$, thus every type realized in $M$ is recursive in some $s \in S$. Let $\Gamma(\bar{v}, \bar{w})$ be a type coded in $\operatorname{Re}(M)$, i.e. $\varphi(\bar{v}, \bar{w}) \in \Gamma(\bar{v}, \bar{w})$ iff $P_{\ulcorner\varphi\urcorner} \mid a$ for some $a \in M$. Then for any $\bar{m} \in M$, $M$ must realize $\left\{\varphi(\bar{v}, \bar{m}) \leftrightarrow P_{\ulcorner\varphi\urcorner} \mid a: \varphi\right.$ an $L$-formula $\}$. Hence $M$ is $\operatorname{Re}(M)$-saturated.

The following lemma is taken from [M].

LEMMA 3.2. If $M \vDash T, d \in D(\nabla, M)$ and $e_{T} \geqslant d$, then $e \in D(\nabla, M)$.

Proof. Let $M=(\omega, \oplus, \ldots) \vDash T$, such that $\oplus, \ldots, \leqslant_{T} d$. Let $f: \omega \rightarrow \omega$ be such that $f \leqslant_{T} e$ and $M \vDash f(m)$ is even iff $m \in d$. For $R\left(x_{1}, \ldots, x_{n}\right)$ a relation in $L$, define $R^{\prime}$ by

$$
R^{\prime}\left(m_{1}, \ldots, m_{n}\right) \text { iff } M \vDash R\left(f\left(m_{1}\right), \ldots, f\left(m_{n}\right)\right) .
$$

Let $N=\left(\omega, \oplus^{\prime}, \odot^{\prime}, \ldots\right)$; clearly $e \in D(\nabla, n)$ and $N \cong M$. Let $\Delta$ be the set of all $L$-formulas.

Corollary 3.3. If $M \vDash T$ is $S$-saturated, then $D(\nabla, M)=D(\Delta, M)=D(S)$. Corollary 2.12 shows that $D(\nabla, M)$ is dense in $D(S)$. Clearly $D(\nabla, M)$ is dense in $D(\Delta, M)$, and we have showed above that $S=\operatorname{Re}(M)$ so $D(S)$ is dense in $D(\nabla, M)$. Equality holds because by 3.2 and 2.3(iii), $D(\nabla, M)$ and $D(S)$ are closed upwards.

We should note that the conclusion $D(\nabla, M)=D(\Delta, M)$ uses both recursive saturation and the coding power of arithmetic. In general, if we know $d \in D(\nabla, \mathfrak{U})$, we can only conclude that $d^{(\omega)} \in D(\Delta, \mathfrak{A})$. Harrington [H] gives an example of a nonstandard $M \vDash \mathbf{P}$ with $0^{\prime} \in D(\nabla, M)$ but $T h(M) \equiv_{T} 0^{(\omega)}$. There are also known examples of nonstandard models of $\operatorname{Th}(\mathbf{N})$ coded by degrees strictly below $0^{(\omega)}$ (see [K2, M, KLS]).

The following example shows that $D(\nabla, \mathfrak{A})=D(\Delta, \mathfrak{U})$ is not a general property of recursively saturated models.

Proposition 3.4. There is a complete undecidable theory $T$ with a recursively presented $\omega$-saturated model $\mathfrak{A}$. (Thus $0 \in D(\nabla, \mathfrak{A})$, but not $D(\Delta, \mathfrak{A})$.)

Proof. If $\{n\}(n) \downarrow$, let $u_{n}=2 s$ where $\{n\}(n)$ halts at exactly step $s$. Let

$$
U_{n}= \begin{cases}\left\{u_{n}\right\} & \text { if }\{n\}(n) \downarrow, \\ \varnothing & \text { otherwise. }\end{cases}
$$

Let $\mathfrak{A}=\left(\omega, U_{n}: n \in \omega\right), L=\left\{U_{i}: i \in \omega\right\} . \operatorname{Th}(\mathfrak{A})$ is undecidable as $\{n\}(n) \downarrow$ iff $\exists v U_{n}(v) \in \operatorname{Th}(\mathfrak{A})$. The predicate $m \in U_{n}$ is recursive uniformly in $m$ and $n$, so $\mathfrak{A}$ is recursively presented.

Suppose $\mathcal{L} F \operatorname{Th}(\mathfrak{A})$. Let $X^{\mathfrak{L}}=\left\{b \in \mathcal{L}\right.$ for some $\left.n, \mathcal{L} \vDash U_{n}(b)\right\}$. If $\mathcal{E} F \operatorname{Th}(\mathfrak{A})$, then $X^{\varrho} \cong X^{\mathfrak{E}}$. Thus the isomorphism type of $\mathcal{L}$ is determined by $\varrho \backslash X^{\mathfrak{E}}$. As no relations hold on $\mathscr{L} \backslash X^{\mathfrak{E}}$, the isomorphism type of $\mathscr{L}$ is determined by $\left|\mathfrak{L} \backslash X^{\mathfrak{E}}\right| .\left|\mathfrak{A} \backslash X^{\mathfrak{A}}\right|=\boldsymbol{\aleph}_{0}$, so $\mathfrak{A}$ is the $\omega$-saturated model. 
(b) Skolem arithmetic. Let $T$ be a complete extension of $\operatorname{Th}(\omega, \cdot)$ in some suitable language. Let $M \vDash T$ and let $b \in M$ be an infinite power of 2. If $a \in M$ and $a \mid b$ we define $r_{b}^{*}(a)=\left\{n: \exists x x^{p_{n}}=a\right\}$ and $\operatorname{Re}_{b}^{*}(M)=\left\{r_{b}^{*}(a): a \mid b\right\}$.

LeMma 3.5. If $d \in D(\nabla, M)$, then, for $a \mid b, r_{b}^{*}(a) \leqslant_{T} d$ uniformly in $a, b$.

Proof. Clearly $r_{b}^{*}(a)$ is r.e. in $d$, so we need only show $\left\{n: \nexists x x^{p_{n}}=a\right\}$ is r.e. in $d$. $a$ is a power of 2 , say $a=2^{m}$. Thus $\nexists x x^{p_{n}}=a$ iff $p_{n}+m$. Thus $\nexists x x^{p_{n}}=a$ iff $\exists x W_{i<p_{n}} x^{p_{n}} 2^{i}=a$. Hence $\left\{n: \nexists x x^{p_{n}}=a\right\}$ is r.e. in $d$.

Lemma 3.6. $T$ is effectively perfect and if $M \vDash T$ is $S$-saturated, then $S=\operatorname{Re}_{b}^{*}(M)$, where $b$ is any nonstandard power of 2.

Proof. The function $\varphi(\alpha)=\mathbb{X}\left\{\exists x x^{p_{n}}=v: \alpha(n)=1\right\} \wedge \bigwedge\left\{\not \nexists x x^{p_{n}}=v\right.$ : $\alpha(n)=0\}$ effectively demonstrates non $\omega$-stability. The second part of the proof is done as in Lemma 3.1.

We define, for recursively saturated $M \vDash T, \operatorname{Re}^{*}(M)$ as $\operatorname{Re}_{b}^{*}(M)$, for any $b$ a nonstandard power of 2 .

Lemma 3.7. If $M \vDash T$ is $S$-saturated, then $D(S)$ is dense in $D(\nabla, M)$.

Proof. Let $d \in D(\nabla, M)$. Fix a listing $m_{0}, m_{1}, \ldots$ of $M$. We begin dovetailing searches for $x$ such that $x m_{i}=b$ and list as $n_{0}, n_{1}, \ldots$, all $m_{i}$ for which such an $x$ is found. This is $d$-effective. Let

$$
g(i, j)= \begin{cases}1 & \text { if } j \in r^{*}\left(n_{i}\right) \\ 0 & \text { otherwise. }\end{cases}
$$

$g \leqslant_{T} d$, since the decision $j \in r^{*}\left(n_{i}\right)$ is $d$-recursive uniformly in $i$ and $j$. By Lemma 3.6, $g$ is an enumeration of $S$.

We need an analog of Lemma 3.2 for multiplication. This padding argument was provided for us by Julia Knight.

Lemma 3.8. Let $M=(\omega, \odot)=\operatorname{Th}(\omega, \cdot)$. Let $a \in M$ be an infinite power of 2 and let $b \in M$ be an infinite power of 3. If $e_{T} \geqslant \odot$, then $e \in D(\omega, M)$.

Proof. We define $f: \omega \rightarrow \omega$ a bijection recursive in $e$ such that:

(i) if $n \in e, M \vDash f(2 n) \mid a$,

(ii) if $n \notin e, M \vDash f(2 n) \mid b$.

Let $n$ be the structure induced on $\omega$ by $f$. Clearly $\{n: n \in e\}$ and $\{n: n \notin e\}$ are both r.e. in the diagram of $N$. As $f \leqslant_{T} e, \odot_{n} \leqslant_{T} e$.

The result still holds if we drop the assumptions of the existence of $a$ and $b$; however, as we consider only recursively saturated models, this assumption is harmless.

Corollary 3.9. If $M$ is $S$-saturated, then $D(\nabla, M)=D(\Delta, M)=D(S)$.

Proof. The proof is clear from Lemmas 3.7, 3.8 and Corollary 2.12.

(c) Subsystems of PA.I $\Sigma_{n}$ will denote the subsystem of PA obtained by restricting the induction schema to $\Sigma_{n}$ formulas. EXP will denote $I \Sigma_{0}+\forall x, y \exists z x^{y}=z$, where this is suitably formalized. It can be shown that $\left(I \Sigma_{1} \supsetneqq \operatorname{EXP} \supsetneqq I \Sigma_{0}\right)$. If $M$ is 
a $\{+, \cdot, 0,1\}$-structure, $M_{+}$will denote the reduct of $M$ to $\{+, 0,1\}$ and $M$. the reduct to $\{\cdot, 0,1\}$. If $\Gamma$ is a set of formulas, $\Gamma_{+}$and $\Gamma$. are then restrictions to formulas in the language $\{+, 0,1\}$ and $\{\cdot, 0,1\}$, respectively. $\Delta$ is the set of all $\{+, \cdot, 0,1\}$ formulas, while $\nabla$ is the set of basic formulas.

Corollary 3.10. If $M \vDash I \Sigma_{0}$ is $S$-saturated, then $S=\operatorname{Re}(M)=\operatorname{Re}^{*}(M)$. Furthermore, $D\left(\nabla_{+}, M_{+}\right)=D\left(\Delta_{+}, M_{+}\right)=D(\nabla, M)=D(\Delta, M)=D(S)$ and $D(S)$ is dense in $D\left(\nabla ., M_{.}\right)$.

Proof. This follows from Corollary 3.3 and Lemma 3.7.

Some of our results may be extended to models which are not recursively saturated. We will require the following results from the literature.

THEOREM 3.11 (BARWISE-SCHLIPF [Sch]). Let $M$ be a recursively saturated L-structure. Let $T$ be a recursive $L\left(R_{1}, \ldots, R_{n}\right)$ theory. If $T \cup \mathrm{Th}(M)$ is consistent, then there are $X_{1}, \ldots, X_{n} \subseteq M$ such that $M\left(X_{1}, \ldots, X_{n}\right)$ is a recursively saturated model of $T$.

COROllaRY 3.12. If $M=\operatorname{Th}(\omega,+)$ (or $M=\mathrm{Th}(\omega, \cdot))$ is recursively saturated and $T \in \operatorname{Re}(M)$ is a completion of $\mathbf{P}$, then $M$ has an expansion to a recursively saturated model of $T$.

Proof. Let $t \in M$ such that $r(t)=T$. Let $T^{*}=\left\{\varphi \leftrightarrow p_{\ulcorner\varphi\urcorner} \mid t: \varphi\right.$ a sentence $\}$ and apply Corollary 3.11 .

Theorem 3.13 (Cegielski, McAloon And Wilmers [CMW]). (i) If $M$ is $a$ nonstandard model of $I \Sigma_{0}$, then $M_{+}$is recursively saturated.

(ii) If $M$ is a nonstandard model of EXP, then $M$. is recursively saturated.

Theorem 3.13 extends results of Lipschitz and Nadel [LN] for P. Cegielski, McAloon and Wilmers point out that EXP cannot be weakened to $I \Sigma_{0}$ in (ii).

LEMMA 3.14. If $M \vDash I \Sigma_{0}$, then $\operatorname{Re}(M)=\operatorname{Re}^{*}(M)$.

Proof. Let $b \in M$ be the infinite power of 2 we use for coding. Let $r(a) \in \operatorname{Re}(M)$. For $n \in \omega, M \vDash \exists v \leqslant a\left(\forall m<n\left(p_{m} \mid v \leftrightarrow\left(\exists z<v z^{p_{m}}=v\right)\right) \wedge v \mid b\right)$. By $\Sigma_{0}$-overspill this must also hold for some nonstandard $N$. If $c$ witnesses this, then $r(a)=$ $r^{*}(c)$. Thus $\operatorname{Re}(M) \subseteq \operatorname{Re}^{*}(M)$. The proof of the reverse inclusion is similar.

Corollary 3.15. If $M \vDash I \Sigma_{0}$, then $D\left(\nabla_{+}, M\right)=D\left(\Delta_{+}, M_{+}\right)=D(\operatorname{Re}(M))$.

PROOF. The proof is clear from Corollary 3.3 and Theorem 3.13.

Corollary 3.16. If $M \vDash \operatorname{EXP}$, then $D(\operatorname{Re}(M))$ is dense in $D\left(\nabla ., M_{.}\right)$.

Proof. The proof is clear from Lemma 3.14, Theorem 3.13, and Lemmas 3.7 and 3.8 .

Corollary 3.17. Let $M$ F EXP. Then $D\left(\Delta_{.}, M_{*}\right)$ is dense in $D\left(\nabla_{+}, M_{+}\right)$and $D\left(\Delta_{+}, M_{+}\right) \supseteq D\left(\nabla ., M_{\text {. }}\right)$.

Proof. Let $d_{0}$ code the basic diagram of $M_{+}$and $d_{1}$ code the basic diagram of $M$. . Let $T$ be a complete extension of PA coded in $\operatorname{Re}(M)$. By 3.12 we can expand 
$M_{+}$and $M$. to $N=\left(M_{+}, \odot\right)$ and $N^{*}=\left(M_{.}, \oplus\right)$ recursively saturated models of $T$. By $3.9, N$ and $N^{*}$ are $\operatorname{Re}(M)$-saturated so $N \cong N^{*}$. Thus $N_{\odot} \cong M$. and $N_{\oplus}^{*} \cong M_{+}$. By Corollary 2.12 we can present $N$ and $N^{*}$ with full diagrams recursive in $d_{0}$ and $d_{1}$, respectively. Thus $D\left(\Delta_{.}, M_{*}\right)$ is dense in $D\left(\nabla_{+}, M_{+}\right)$and $D\left(\nabla_{+}, M_{+}\right)$is dense in $D\left(\Delta_{.}, M_{\text {. }}\right)$. By Lemma 3.2, $D\left(\nabla_{+}, M_{+}\right) \supseteq D\left(\nabla ., M_{\text {. }}\right)$.

(d) Initial segments. Let $M=I \Sigma_{0}$. Let $a$ be a nonstandard element of $M$. We define a structure $[0, a]$ with universe $\{x \in M: x \leqslant a\}$ and functions + and . defined by

$$
\begin{aligned}
& x+y= \begin{cases}z & \text { if } z<a \text { and } M=x+y=z, \\
a & \text { if } M=x+y \geqslant a ;\end{cases} \\
& x \cdot y= \begin{cases}z & \text { if } z<a \text { and } M \vDash x \cdot y=z, \\
a & \text { if } M \vDash x \cdot y \geqslant a .\end{cases}
\end{aligned}
$$

For $b \in[0, a]$ we define $r_{*}(b)=\left\{n: M \vDash p_{n} \mid b\right\}$.

Lemma 3.18. $\operatorname{Re}(M)=\left\{r_{*}(b): b<a\right\}$ and $D(\operatorname{Re}(M)) \supseteq D(\nabla,[0, a])$.

Proof. The proof is similar to Lemma 3.14 and Corollary 3.3.

Lemma 3.19. $D(\nabla,[0, a])$ is closed upward in the Turing degrees.

Proof. The proof is similar to Lemma 3.2.

Corollary 3.20. If $M \vDash I \Sigma_{0}$ and $[0, a]$ is $\operatorname{Re}(M)$-saturated, then $D(\nabla,[0, a])=$ $D(\Delta,[0, a])=D(\operatorname{Re}(M))$.

The following result shows that the hypothesis of Corollary 3.20 holds reasonably often.

LEMMA 3.21 (LESSAN [L]). Let $M$ F EXP. Then $[0, a]$ is recursively saturated.

The techniques of [CMW] can be used to find models of $I \Sigma_{0}$ such that $[0, a]$ is not recursively saturated.

COROllary 3.22. If $M \vDash$ EXP and $a$ and $b \in M$ are nonstandard, then $D(\nabla,[0, a])=D(\nabla,[0, b])$.

Proof. Using Theorem 3.11 and Lemma 3.21 we can find $N \vDash$ EXP such that $[0, a] \subseteq_{e} N$ and $[0, b] \subseteq_{e} N$ such that $N$ is recursively saturated. (If $M \vDash I \Sigma_{1}$, we could use the indicators of Kirby, McAloon and Murawski [KMM] to choose $N \subseteq \subseteq_{e}$.) We then apply Lemma 3.21.

Stronger results are available if we look at $[0, a]_{+}$, the reduct of $[0, a]$ to addition.

Lemma 3.23 (CEgielski, MCAloon AND Wilmers [CMW]). If $M \vDash I \Sigma_{0}$, then $[0, a]_{+}$is recursively saturated.

COROLlaRY 3.24. If $M \vDash I \Sigma_{0}$ and $a, b \in M$ are nonstandard, then $D\left(\nabla_{+},[0, a]_{+}\right)$ $=D\left(\nabla_{+},[0, b]_{+}\right)$. 
Proof. The proof is as in Corollary 3.22.

(e) Residue fields. Suppose $M$ F PA, and $M \vDash p$ is prime. Then $M / p$ is a field of characteristic 0 if $p$ is infinite. Since " $M$ thinks $M / p$ is finite", $M / p$ is recursively saturated [Mac2]. If $p$ is infinite $M / p$ is pseudofinite in the sense of $\mathrm{Ax}[\mathbf{A}]$; and, in fact, every countable recursively saturated pseudofinite $K$ of characteristic 0 is of the form $M / p$ for suitable $M, p$ [Mac2].

It is (essentially) proved in [Mac1] that if $T$ is a complete theory of pseudofinite fields of characteristic 0 then $T$ is effectively perfect. The appropriate map on $2^{\omega}$ is given by

$$
\begin{aligned}
\varphi(\alpha)= & \mathbb{X}\left\{(\exists x)\left(v+n=x^{2}\right): \alpha(n)=1\right\} \\
& \wedge \mathbb{X}\left\{\neg(\exists x)\left(v+n=x^{2}\right): \alpha(n)=0\right\} .
\end{aligned}
$$

Suppose $K \vDash T$. Let $\delta$ be a nonsquare in $K$. Then $\left\{\neg(\exists x)\left(v+n=x^{2}\right)\right\}$ is equivalent (modulo $T)$ to $(\exists x)\left(v+n=\delta \cdot x^{2}\right)$. This fact should recall 3(a).

Suppose $a \in K$. We define $r_{K}(a)$ as $\left\{n \in \omega:(\exists x)\left(a+n=x^{2}\right)\right\} . \operatorname{Re}(K)$ is defined as $\left\{r_{K}(a): a \in K\right\}$.

Let $\nabla$ be the set of basic formulas of the language of field theory, and $\Delta$ the set of all formulas of field theory. Using the observation of the penultimate paragraph, one easily shows the following:

If $d$ is the $\nabla$-degree of $K^{f}$, then $r_{K}(f(i))$ is recursive in $d$, uniformly in $i$.

The obvious analog of Lemma 3.1 is

Lemma 3.25. If $K$ is recursively saturated, and $S$ is the Scott set of $K$, then $S=\operatorname{Re}(K)$.

PROOF. The proof is clear.

Now the analog of Lemma 3.2:

Lemma 3.26. If $d \in D(\nabla, K)$ and $e \geqslant d$ then $e \in D(\nabla, K)$.

Proof. If one examines the proof of Lemma 3.2, one sees that all one needed was an infinite coinfinite set which was both $\Sigma$ - and $\Pi$-definable, perhaps using parameters. In Lemma 3.2 one took the set of even elements. Now one takes the squares.

Corollary 3.27. If $K$ is $S$-saturated, then $D(\nabla, K)=D(\Delta, K)=D(S)$.

PROof. The proof is as for Corollary 3.3.

Now suppose $K$ is $M / p$, where $M$ F PA and $p$ is an infinite prime. Define $S(p)$ as the Scott set of $M / p$. Let $S$ be the Scott set of $M$.

Proposition 3.28. $S=S(p)$.

Proof. Clearly $S(p) \subseteq S$. Now suppose $r \in S$. Consider the (partial) type

$$
r_{M / p}(v)=r \text {. }
$$

Precisely, fix $a$ so that $r=\left\{n: p_{n} \mid a\right\}$, and consider $\{(n+v$ is a quadratic residue $\left.\bmod p) \leftrightarrow p_{n} \mid a: n \in \omega\right\}$. This is of bounded complexity, and finitely satisfiable in $M$, so satisfiable. So $r \in S(p)$. 
Corollary 3.29. $D(\nabla, M / p)=D(\Delta, M / p)=D(S)$.

PROOF. The proof is clear.

ReMARKs. (1) Let $\oplus_{p}, \odot_{p}$ be, respectively, the addition and multiplication in $M / p$. $\oplus_{p}$ is a divisible abelian group, and is in fact $Q^{(\omega)}$. It follows easily that $D\left(\oplus_{p}\right)$ is the set of all Turing degrees, because of the following

Proposition 3.30. $Q^{(\omega)}$ has presentations of each degree.

Proof. Fix $b$ a nonzero element of $\mathbf{Q}^{(\omega)}$ and write $\mathbf{Q}^{(\omega)}=\langle b\rangle \oplus D$, where $D \cong \mathbf{Q}^{(\omega)}$. $D$ has a recursive presentation $g$. Any $x \in \mathbf{Q}^{(\omega)}$ has a unique representation $x=r \cdot b+d$ where $d \in D$ and $r \in \mathbf{Q}$.

Let $h$ : $\mathbf{Q} \rightarrow \omega$ be a bijection. We define a presentation $f$ of $\mathbf{Q}^{(\omega)}$ by $f(r \cdot b+d)=$ $\langle h(r), g(d)\rangle$, where $\langle$,$\rangle is some fixed recursive pairing function. For i=0,1,2$ let $f\left(r_{i} \cdot b+d_{i}\right)=n_{i}$. Then $\left(\mathbf{Q}^{(\omega)}\right) \vDash n_{0}+n_{1}=n_{2}$ iff $d_{0}+d_{1}=d_{2}$ and $r_{0}+r_{1}+r_{2}$, and this is decidable from $h$.

On the other hand,

$$
h\left(\frac{m}{n}\right)=k \Leftrightarrow\left(\mathbf{Q}^{(\omega)}\right)^{f} \vDash \frac{m}{n} f(b)=\langle k, g(0)\rangle \Leftrightarrow\left(\mathbf{Q}^{(\omega)}\right)^{f} \vDash m f(b)=n\langle k, g(0)\rangle .
$$

Thus given the diagram of $\left(\mathbf{Q}^{(\omega)}\right)^{f}$ we may compute $h$. Thus $\left(\mathbf{Q}^{(\omega)}\right)^{f} \equiv_{T} h$. As $h$ may be chosen to have arbitrary degree, $D\left(\oplus_{p}\right)=$ all Turing degrees.

With $\odot_{p}$ the situation is more delicate. In any $M$ F PA there are $p$ such that $D\left(\odot_{p}\right)$ is the set of all Turing degrees. But also there are, in $M, p$ such that $D\left(\odot_{p}\right)$ is not the set of all Turing degrees. One can prove the following precise result.

Proposition 3.31. $D\left(\odot_{p}\right)=\left\{d: d \geqslant\right.$ the degree of $\left.\operatorname{Th}\left(\odot_{p}\right)\right\}$.

Proofs of these claims about $\odot$ would take us too far afield.

(2) There is some interest in knowing what fragment of PA suffices for the results on $M / p . I \Sigma_{1}$ is certainly enough, and it is reasonable to conjecture that EXP is enough. With respect to $I \Sigma_{0}$, we cannot now exclude the possibility that $M / p$ may be algebraically closed!

(f) The real fields of $M$. Let $M$ F PA. Let $Q(M)$ be the field of fractions of $M$. For $1 \leqslant n<\omega$ we consider the Dedekind cuts in $\mathbf{Q}(M)$ which are $\Sigma_{n}$-definable (say in $M$, via pairing). Let $\mathbf{R}^{\Sigma_{n}}(M)$ be the set of $\Sigma_{n}$-definable Dedekind cuts, endowed with the obvious definable addition and multiplication. It is easily proved that $\mathbf{R}^{\Sigma_{n}}(M)$ is real closed. The theory of real closed fields is effectively perfect (and as in (e) this can be witnessed by $\Sigma \wedge \Pi$ formulas [Mac1]). One shows easily that $\mathbf{R}^{\Sigma_{n}}(M)$ is recursively saturated (use quantifier-elimination).

Let $S^{n}$ be the Scott set of $\mathbf{R}^{\Sigma_{n}}(M)$.

Proposition 3.32. (a) $S^{\Sigma_{n}}=S(M)$.

(b) $D\left(\nabla, \mathbf{R}^{\Sigma_{n}}(M)\right)=D\left(\Delta, \mathbf{R}^{\Sigma_{n}}(M)\right)=D(S(M))$.

CoROLlaRY 3.33. $\mathbf{R}^{\Sigma_{n}}(M) \cong \mathbf{R}^{\Sigma_{m}}(M)$.

The fields $\mathbf{R}^{\Sigma_{n}}(M)$ are important for effective estimates in nonstandard analysis [Mac3]. In this connection it is amusing to construct $S(M)$ in more algebraic terms. 
Namely, we consider the $\mathbf{R}^{\boldsymbol{\Sigma}_{n}}$ valuation ring $\mathbf{B}^{\boldsymbol{\Sigma}_{n}}$ of elements bounded by $\mathbf{Z}$. In $\mathbf{B}^{\boldsymbol{\Sigma}_{n}}$ we have the ideal of nonunits $I^{\Sigma_{n}}$. $\mathbf{B}^{\Sigma_{n}} / I^{\Sigma_{n}}$ is canonically embeddable in $\mathbf{R}$, and its image is $S(M)$. So $S$ is identified as a set of monads.

4. Classification results. Let $T$ be a complete extension of $\mathbf{P}$. What degrees code nonstandard models of $T$ ? It is an interesting open problem to classify the degrees coding nonstandard models of $T$. In this section we present classifications of degrees coding various related structures. The easiest result is the following.

COROLlARY 4.1. $d$ codes a recursively saturated model of $T$ iff $d$ enumerates a Scott set containing $T$.

Proof. The proof is clear from Lemma 1.3(i) and Corollary 2.12.

COROLLARY 4.2. $d$ codes a recursively saturated model of $\operatorname{Th}(N)$ iff $d$ enumerates a Scott set containing $0^{(\omega)}$.

Our main tool is the following theorem of Knight [K1].

THEOREM 4.3 (KNIGHT [K1]). Let $S$ be a countable Scott set. $S$ occurs as $\operatorname{Re}(M)$ for some $M \vDash T$ iff $T \cap \Sigma_{n}^{0} \in S$ for any $n$.

Corollary 4.4 (KNIGHT [K1]). Let $M \vDash \mathrm{Th}(\omega,+)$. $M$ can be expanded to a model of $T$ iff, for any $n, T \cap \Sigma_{n}^{0} \in S$ and $M$ is recursively saturated.

COROLlaRY 4.5. $d$ codes the addition of a model of $T$ iff $d$ enumerates a Scott set containing all $T \cap \Sigma_{n}^{0}$.

Proof. $(\Rightarrow)$ is clear.

$(\Leftarrow)$ Let $d$ enumerate $S$. By Corollary 2.12 we can find $N \vDash \operatorname{Th}(\omega,+)$ with $\operatorname{Re}(N)=S$ such that $N$ is recursively saturated and $N$ is coded by $d$. But if $M$ is any model of $T$ with $\operatorname{Re}(M)=S, M_{+} \cong N$. So $d$ codes the addition of a model of $T$.

Related results hold for $\operatorname{Th}(\omega, \cdot)$.

Corollary 4.6. (i) Let $M \vDash \operatorname{Th}(\omega, \cdot) . M$ can be expanded to a model of $T$ iff $M$ is recursively saturated and every $T \cap \Sigma_{n}^{0} \in \operatorname{Re}(M)$.

(ii) $d$ codes the multiplication of a model of $T$ iff $d$ enumerates a Scott set containing each $T \cap \Sigma_{n}^{0}$.

Proof. The proof is as above.

Using the following result we can classify degrees coding initial segments and residue fields.

TheOREM 4.7 (FRIEDMAN). Let $M$ and $N$ be countable models of P. $M$ can be embedded as an initial segment of $N$ iff $\operatorname{Re}(M)=\operatorname{Re}(N)$ and $\operatorname{Th}_{\Sigma_{1}^{0}}(M) \subseteq \operatorname{Th}_{\Sigma_{1}^{0}}(N)$.

Proof. See [Sm].

COROLlaRy 4.8. $d$ codes $a[0, a]$ where $a \in M, M \vDash T$ iff $d$ enumerates $a$ Scott set containing each $T \cap \Sigma_{n}^{0}$. 
Proof. $(\Leftarrow)$ is clear.

$(\Rightarrow)$ Let $d$ enumerate $S$. Let $T^{*} \in S$ be a completion of PA $\cup\left(T \cap \Sigma_{1}^{0}\right)$. Let $N$ be a recursively saturated model of $T^{*}$ with $\operatorname{Re}(N)=S$ such that $N$ is coded by $d$. By Theorem 4.7 there is an $M_{e} \supseteq N$ such that $M \vDash T$. Thus there is an $a \in N,[0, a]$ is coded by $d$, and $[0, a]$ is an initial segment of a model of $T$.

COROllary 4.9. $d$ codes some $M / p$ where $M \vDash T$ and $p \in M$ is a nonstandard prime iff d enumerates a Scott set such that each $T \cap \Sigma_{n}^{0} \in S$.

Proof. The proof is as in Corollary 4.8.

Definition 4.10. $d$ effectively enumerates a Scott set $S$ iff there are $E \subseteq \omega^{2}, f_{1}$ : $\omega \times \omega \rightarrow \omega, f_{2}: \omega \times \omega \rightarrow \omega$ and $f_{3}: \omega \rightarrow \omega$ such that:

(i) $E, f_{1}, f_{2}, f_{3}$ are recursive in $d$,

(ii) if $E_{i}$ denotes $\{j:(i, j) \in E\}$, then $S=\left\{E_{i}: i \in \omega\right\}$,

(iii) $E_{f_{1}(i, j)}=E_{i} \oplus E_{j}$,

(iv) if $A \subseteq \omega, A \leqslant_{T} E_{i}$ and $\{e\}^{E_{i}}$ is the characteristic function of $A$, then $E_{f(e, i)}=A$,

(v) if $E_{i}$ codes an infinite binary tree, then $E_{f_{3}(i)}$ codes an infinite path through $T$.

Solovay has proved the following characterization of degrees coding models of $\operatorname{Th}(N)$.

TheOREM 4.11 (Solovay [So]). $d$ codes a nonstandard model of Th( $N)$ with Scott set $S$ iff $d$ effectively enumerates $S$.

We would like to show that $d$ effectively enumerates $S$ iff $d$ enumerates $S$. To do this we must first examine the easier direction of Solovay's theorem.

Lemma 4.12 (Solovay [So]). If $d$ codes a nonstandard $M \vDash \mathbf{P}$, then $d$ effectively enumerates $\operatorname{Re}(M)$.

Proof. Let $M=(\omega, \oplus, \odot)$. Let $E=\left\{(i, j): M \vDash p_{j} \mid i\right\}$ where $E$ is an enumeration of $\operatorname{Re}(M)$ recursive in $d$. We must define $f_{1}, f_{2}, f_{3}$. Fix $n \in M$ a nonstandard element:

(i) Let $a, b \in M$. Let $\sigma: M \rightarrow M^{2}$ be the usual coding function. We know that $M \vDash \exists v\left(v>N \wedge \forall m<v\left(p_{m} \mid v \leftrightarrow\left(p_{\sigma_{0}(m)}\left|a \wedge p_{\sigma_{1}(m)}\right| b\right)\right)\right.$. By Matijasevič's theorem this sentence can be written in the form $\exists v \varphi(v, a, b)$, where $\varphi$ is existential and independent of our choice of $a$ and $b$. Let $\varphi(v, a, b)$ be $\exists w \psi(w, v, a, b)$ where $\psi$ is quantifier free. To calculate $f_{1}(a, b)$ we enumerate all pairs $\langle n, m\rangle$ and find the first pair $\langle c, w\rangle$ such that $\psi(w, c, a, b)$ holds. Let $f_{1}(a, b)=c$.

(ii) Suppose $\{e\}^{E_{a}}$ is total. We know that $M \vDash \exists v\left(v>N \wedge \forall m<n\left(p_{m} \mid v \leftrightarrow\right.\right.$ $\{e\}^{\left\{b \in M: M=p_{b} k\right\}}(m)$ converges within $N$ steps and outputs 1.)). Again we can use Matijasevič's theorem to rewrite this as an existential formula and may compute $f_{2}$ by searching for instantiations.

(iii) Suppose $E_{a}$ is an infinite binary tree. $M$ believes there is a $b \in X=\{b$ : $\left.M \vDash p_{b} \mid a\right\}$ such that $b$ codes a binary sequence $\alpha$ such that every subsequence of $\alpha$ is $X$ and $\alpha$ is of maximal length for sequences with this property. To calculate $f_{3}$, we use Matijasevič's theorem to allow us to effectively search for $b$ and $c$, where $b$ is as above and $\forall n<c p_{n} \mid c \leftrightarrow \alpha(n)=1$. 
Corollary 4.13. If $d$ enumerates $S$, then $d$ effectively enumerates $S$.

Proof. Let $T \in S$ be a complete extension of PA. By Corollary 2.12 there is a recursively saturated $N \vDash T$ such that $\operatorname{Re}(N)=S$ and $N$ is coded by $d$. But then by Lemma 4.12, $d$ effectively enumerates $S$.

COROllary 4.14. $d$ codes a model $M$ of $\operatorname{Th}(N)$ with $\operatorname{Re}(M)=S$ iff, for all $n$, $0^{(n)} \in S$ and $d$ enumerates $S$.

COROllary 4.15. There is a nonstandard $M \vDash \mathrm{Th}(N)$ and $d$ coding $M$ such that $\operatorname{Re}(M)=\{X \subseteq \omega: X$ is arithmetic $\}$ and $d^{\prime \prime}=0^{(\omega)}$.

Proof. Hodes [Ho] has shown that there are $d$ enumerating the arithmetic degrees such that $d^{\prime \prime}=0^{(\omega)}$. The result now follows from Corollary 4.14.

Corollary 4.15 generalizes the existence results in [KLS].

\section{REFERENCES}

[A] J. Ax, The elementary theory of finite fields, Ann. of Math. (2) 88 (1968).

[CMW] P. Cegielski, K. McAloon and G. Wilmers, Modeles recursivement satures de l'addition et de la multiplication des entiers naturels, preprint.

[G] S. S. Goncharov, Strong constructivizability of homogeneous models, Algebra i Logika 17 (1978).

$[\mathbf{H}]$ L. Harrington, Building arithmetical models of $P A$, handwritten notes, Berkeley, 1979.

[Ho] H. Hodes, Uniform upper bounds on ideals of Turing degrees, J. Symbolic Logic 43 (1978).

[KMM] L. Kirby, K. McAloon and R. Murawski, Indicators, recursive saturation and expandability, Fund. Math. 114 (1982).

[K1] J. Knight, Additive structure in uncountable models for a fixed completion of $P$, J. Symbolic Logic (to appear).

[K2] _ A nonstandard model of arithmetic of degree less than that of true arithmetic, handwritten notes, Notre Dame, 1978.

[KLS] J. Knight, A. Lachlan and R. Soare, Two theorems on degrees of models of true arithmetic, preprint.

[KN1] J. Knight and M. Nadel, Expansions of models and Turing degrees, J. Symbolic Logic 47 (1982).

[KN2] , Models of arithmetic and closed ideals, J. Symbolic Logic (to appear)

[L] H. Lessan, Models of arithmetic, Ph.D. thesis, Manchester University, 1978.

[LN] L. Lipschitz and M. Nadel, The additive structure of models of PA, Proc. Amer. Math. Soc. 68 (1978).

[Mac1] A. Macintyre, The complexity of types in field theory, Logic Year 1979-80 (M Lerman, J. Schmerl and R. Soare, eds.), Springer-Verlag, Berlin and New York, 1980.

[Mac2] - Residue fields of models of $P$, Logic, Methodology and Philosophy of Science. VI (Hannover 1979) (L. Cohen, J. Los, H. Pfeiffer and K. Podewski, eds.), North-Holland, Amsterdam, 1982.

[Mac3] _ Nonstandard analysis and effective estimates, in preparation.

[M] D. Marker, Degrees of models of true arithmetic, Proceedings of the Herbrand Symposium (J. Stern, ed.), North-Holland, Amsterdam, 1982.

[Mi] T. Millar, Foundations of recursive model theory, Ann. Math. Logic 13 (1978).

[Mo] M. Morley, Decidable models, Israel J. Math. 25 (1976).

[N] M. Nadel, On a problem of MacDowell and Specker, J. Symbolic Logic 45 (1980).

[P] M. G. Peretyat'kin, Criterion for strong constructivizability of a homogeneous model, Algebra i Logika 17 (1978).

[Sch] J. Schlipf, A guide to the identification of admissible sets over structures, Ann. Math. Logic 12 (1978).

[S] D. Scott, Algebras of sets binumerable in complete extensions of arithmetic, Proc. Sympos. Pure Math., vol. 5, Amer. Math. Soc., Providence, R.I., 1962.

[Sm] C. Smorynski, Recursively saturated nonstandard models of arithmetic, J. Symbolic Logic 45 (1981).

[So] R. Solovay, personal communication, 1982. 
[T] S. Tennenbaum, Nonarchimedean models of arithmetic, Notices Amer. Math. Soc. 6 (1979).

[W1] G. Wilmers, Ph.D. thesis, Oxford, 1975.

[W2] _ Minimally saturated models, Model Theory of Algebra and Arithmetic (Karpacz 1979) (L. Pacholski, J. Wierzejewski and A. J. Wilkie, eds.), Springer-Verlag, Berlin and New York, 1981.

Department of Mathematics, Yale University, New Haven, Connecticut 06520 (Current address of Angus Macintyre)

Current address (David Marker): Department of Mathematics, University of California, Berkeley, California 94720 